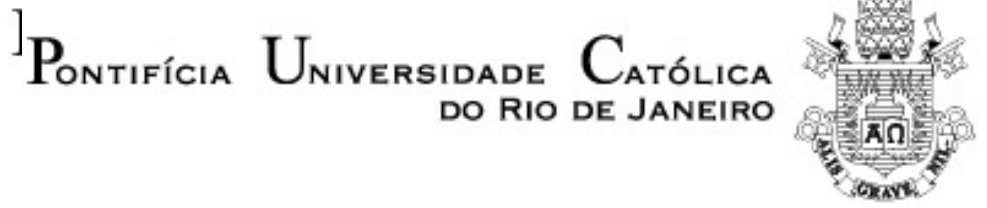

Danilo Marcondes de Souza Neto

“A política brasileira para as operações de paz e intervenções humanitárias: normas, ética e regionalização no envolvimento brasileiro na MINUSTAH"

Dissertação apresentada como requisito parcial para obtenção do grau de Mestre em Relações Internacionais pelo Programa de Pós-graduação em Relações Internacionais da PUC-Rio.

Orientadora: Profa. Mônica Herz

Rio de Janeiro Março de 2010 


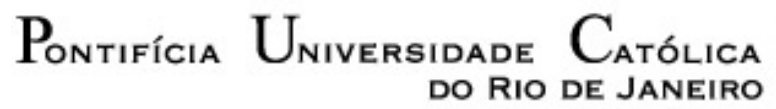

DO RIO DE JANEIRO

Danilo Marcondes de Souza Neto

\section{"A política brasileira para as operações de paz e intervenções humanitárias: normas, ética e regionalização no envolvimento brasileiro na MINUSTAH"}

Dissertação apresentada como requisito parcial para obtenção do grau de Mestre em Relações Internacionais pelo Programa de Pós-graduação em Relações Internacionais da PUC-Rio. Aprovada pela Comissão Examinadora abaixo assinada.

Profa. Mônica Herz

Orientadora

Instituto de Relações Internacionais - PUC-Rio

Prof. Kai Michael Kenkel

Instituto de Relações Internacionais - PUC-Rio

Prof. Antonio Jorge Ramalho da Rocha

Universidade de Brasília - UnB

Profa. Mônica Herz

Vice-Decana de Pós-graduação do Centro de

Ciências Sociais - PUC-Rio

Rio de Janeiro, 26 de março de 2010 
Todos os direitos reservados. É proibida a reprodução total ou parcial do trabalho sem autorização da universidade, do autor e da orientadora.

\section{Danilo Marcondes de Souza Neto}

Graduou-se em Relações Internacionais pela PUC-Rio em 2007. Cursou um semestre de intercâmbio acadêmico na State University of New York at New Paltz (SUNY New Paltz). Foi estagiário do Centro de Informação das Nações Unidas no Rio de Janeiro (UNIC-Rio) e assistente de pesquisa e bolsista de iniciação científica no Instituto de Relações Internacionais da PUC-Rio e no Radar do Sistema Internacional. Atualmente é professor do curso de graduação em Relações Internacionais da PUC-Rio.

\section{Ficha Catalográfica}

Souza Neto, Danilo Marcondes de

A política brasileira para as operações de paz e intervenções humanitárias: normas, ética e regionalização no envolvimento brasileiro na MINUSTAH / Danilo Marcondes de Souza Neto ; orientadora: Mônica Herz. - 2010.

357 f. ; $30 \mathrm{~cm}$

Dissertação (Mestrado) - Pontifícia Universidade Católica do Rio de Janeiro, Instituto de Relações Internacionais, 2010.

Inclui bibliografia 
Aos meus pais e Paula, com todo amor. Às vítimas do terremoto de 12 de janeiro de 2010 no Haiti. 


\section{Agradecimentos}

Gostaria de agradecer à CAPES pelo auxílio concedido, que foi fundamental para que eu pudesse me dedicar de maneira integral à elaboração desta dissertação. Agradeço também à Vice-Reitoria Acadêmica da PUC-Rio pela isenção concedida durante os dois anos de mestrado.

À professora Mônica Herz, agradeço a orientação precisa, o carinho e a amizade desde os tempos em que me orientou na minha monografia de graduação. A professora Mônica tem sido para mim ao longo desses anos de convivência e troca de ideias um exemplo de profissional e pesquisadora da mais alta qualidade. Espero que esta dissertação esteja à altura da competência da sua orientação.

Ao professor Kai Michael Kenkel, agradeço a oportunidade de tê-lo na banca, e também por ter transmitido todos seus valiosos conhecimentos sobre Responsabilidade de Proteger e operações de paz ao longo desses dois anos de mestrado. Agradeço também pela confiança conferida a mim quando fui seu estagiário docente e pelas lições sobre a arte de dar aulas no mesmo período.

Ao professor Antonio Jorge Ramalho, agradeço a oportunidade de tê-lo na minha banca. Suas contribuições sobre o Haiti e a participação brasileira foram fundamentais para a realização da pesquisa apresentada aqui. Agradeço também a simpatia e a hospitalidade com que me recebeu em visita ao Centro de Estudos Brasileiros em Porto Príncipe, em junho de 2008, quando na época era seu diretor. 
Agradeço à professora Andrea Ribeiro Hoffmann, pela sua confiança e estímulo, e por ter sido quem primeiro me ofereceu a oportunidade de trabalhar com pesquisa. No Instituto de Relações Internacionais da PUC-Rio não poderia deixar de agradecer a contribuição fundamental à minha formação em Relações Internacionais, dada pelos seguintes professores: Sonia de Camargo, Nizar Messari, João Pontes Nogueira, José Maria Gomez e Letícia Pinheiro.

No mesmo Instituto, agradeço a atenção e a ajuda sempre eficiente de Regina, Vera, Natacha e Gisele. No Núcleo de Pesquisa e Publicação do Instituto, agradeço a Luciana Varanda pela competência e profissionalismo com que coordena as atividades do Núcleo e pelas sugestões sempre apropriadas.

Não poderia deixar de agradecer às Forças Armadas brasileiras e ao Ministério da Defesa. Faço meus agradecimentos às Forças Armadas a dois oficiais que para mim representam o máximo de competência e de dedicação tanto ao Brasil quanto aos ideais das Nações Unidas: ao Coronel Roberto Escoto, que partilhou comigo a experiência de escrever uma dissertação sobre a presença da ONU no Haiti e cuja ajuda para realizar a visita ao Haiti sou eternamente grato; e ao Capitão-de-Mar-eGuerra (FN) Carlos Chagas Viana Braga, um estudioso da cooperação sulamericana na área de defesa e um oficial altamente comprometido com o envolvimento brasileiro no Haiti.

No Centro de Informação das Nações Unidas (UNIC-Rio), gostaria de agradecer ao diretor Giancarlo Summa, a Valéria Schilling, a Evelyn Rocha e a toda a equipe. Minha experiência de estagiário no UNIC-Rio foi fundamental para aumentar meu interesse na atuação das Nações Unidas e nas operações de paz.

Na Fundação Konrad Adenauer, gostaria de agradecer ao Dr. Wilhelm Hofmeister e ao Dr. Peter Fischer-Bollin, pela oportunidade de ter participado das conferências de segurança internacional do Forte de Copacabana e pelo acesso às publicações da biblioteca do centro de estudos da Fundação no Rio. Agradeço a toda equipe do centro de estudos, em especial a Carla Shores e Luis Fernando Blanco. 
No Itamaraty, gostaria de agradecer a ajuda sempre atenciosa do Secretário Gustavo Senechal de Goffredo Junior, também um estudioso do posicionamento brasileiro em relação às normas internacionais, e do Secretário Eduardo Uziel, este último fundamental para que eu tivesse acesso a contatos e fontes indispensáveis.

Cabe destacar que esse trabalho se beneficia das contribuições fundamentais de autores que abordaram os diversos temas presentes na dissertação, entre esses autores agradeço a troca de ideias e destaco as contribuições de Letícia Pinheiro, Maria Regina Soares de Lima, Monica Hirst, Ricardo Seitenfus e Matias Spektor.

Agradeço também ao professor Arturo Sotomayor pelo material enviado e as discussões sobre o envolvimento do Cone Sul em operações de paz durante as suas visitas ao Rio.

Agradeço a colaboração dos funcionários do serviço de solicitação de documentos e informação da Biblioteca Central da PUC-Rio sem cuja ajuda muitas das inúmeras referências citadas nessa dissertação jamais teriam chegado ao meu alcance.

Agradeço também à minha família, meus avôs, Danilo e Yalu; meus tios, Maria Cândida, Renato, Suyan e Gary e minha prima Anais, pelo apoio e carinho de sempre. Agradeço também à família da Paula, Dr. Paulo, Dona Leda e Bruno pelo carinho e apoio.

Por fim, mas de maneira especial, agradeço aos meus pais, Maria Inês e Danilo, pelo apoio e estímulo constantes, e a Paula, por todo seu amor e por ter me acompanhado nessa jornada como mestrando. 


\section{Resumo}

Souza Neto, Danilo Marcondes de; Herz, Mônica . "A política brasileira para as operações de paz e intervenções humanitárias: normas, ética e regionalização no envolvimento brasileiro na MINUSTAH”. Rio de Janeiro, 2010. 357p. Dissertação de Mestrado - Instituto de Relações Internacionais, Pontifícia Universidade Católica do Rio de Janeiro.

O objetivo desta dissertação é compreender o posicionamento brasileiro perante a norma internacional relacionada a intervenções humanitárias. Para tanto, o presente trabalho faz uso das contribuições da teoria construtivista de Relações Internacionais, orientada por normas que analisam o envolvimento brasileiro na atual Missão de Estabilização das Nações Unidas no Haiti (MINUSTAH). O envolvimento brasileiro é entendido a partir da análise da evolução da norma de intervenção e do contexto normativo regional em que o Brasil está inserido. A hipótese defendida é a de que dois aspectos são fundamentais para se entender o envolvimento brasileiro no Haiti: i) a formulação da resposta à crise haitiana de 2004 como uma resposta regional, na qual os países latino-americanos, principalmente os do Cone Sul da América do Sul, exercem um papel de protagonista; ii) a contextualização da resposta brasileira à crise haitiana nos moldes de uma obrigação ético-moral do país, baseada na solidariedade hemisférica e vinculada ao reconhecimento de uma herança cultural comum por meio do princípio da não indiferença. A conclusão sugerida é de que o envolvimento dos países sul-americanos na MINUSTAH faz parte do esforço brasileiro de fortalecimento da América do Sul, e que, além disso, o interesse nacional não é imutável, mas socialmente construído, podendo incluir uma preocupação com a proteção dos direitos humanos de indivíduos localizados além das fronteiras do Estado brasileiro.

\section{Palavras-chave}

Brasil; Haiti; intervenção humanitária; Nações Unidas; normas; MINUSTAH; operações de paz; política externa; Responsabilidade de Proteger. 


\section{Abstract}

Souza Neto, Danilo Marcondes de; Herz, Mônica (Advisor). "Brazilian policy towards peace operations and humanitarian intervention: norms, ethics and regionalization in Brazilian involvement in MINUSTAH". Rio de Janeiro, 2010. 357p. MSc. Dissertation - Instituto de Relações Internacionais, Pontifícia Universidade Católica do Rio de Janeiro.

The purpose of this dissertation is to understand the Brazilian position regarding the international norm on humanitarian intervention. In order to achieve this objective, the norm oriented constructivist theory of International Relations will be applied in the analysis of the current Brazilian involvement in the United Nations Stabilization Mission in Haiti (MINUSTAH). The Brazilian involvement is understood from the perspective of the evolution of the international norm on intervention but also taking into consideration the regional normative context. The hypothesis developed in this dissertation identifies two aspects as fundamental for the understanding of the Brazilian involvement in Haiti: first, the framing of the response to the Haitian crisis of 2004 as a regional response, in which Latin American countries, particularly those of the Southern Cone, have taken the leading role; second, the framing of the Brazilian response as guided by an ethical and moral obligation, based on the idea of hemispheric solidarity and on the recognition that both countries share a common cultural heritage, which leads to the principle of non-indifference. Our conclusion suggests that the current South American involvement in MINUSTAH is part of a Brazilian project of strengthening South America, and also that a country's national interest is not immutable, but socially constructed, and as such it can include a concern for the protection of human rights of those located outside Brazilian borders.

\section{Keywords}

Brazil; Haiti; humanitarian intervention; United Nations; norms; MINUSTAH; peacekeeping; foreign policy; Responsibility to Protect. 


\section{Sumário}

1. Introdução 17

$\begin{array}{ll}1.1 \text { O recorte empírico } & 21\end{array}$

2. Normas: a discussão teórica 23

2.1 Normas: definições e implicações 23

2.2 Normas e a teoria de Relações Internacionais 29

2.2.1 Normas e a teoria construtivista de Relações 33

Internacionais

2.2.1.1 A contribuição construtivista e o estudo das 39

intervenções humanitárias

2.3 Normas e considerações metodológicas $\quad 45$

2.3.1 A escolha por um modelo de ator fechado 45

2.3.2 O uso do process tracing 46

2.4 Justificativa $\quad 49$

2.4.1 Por que Normas? $\quad 49$

2.4.2 Por que as Nações Unidas? $\quad 49$

2.4.3 Por que operações de paz? 50

2.4.4 Por que o Brasil? 51

2.5 Fontes 53

2.5.1 Entrevistas $\quad 56$

3. Normas na prática: intervenções e operações de paz 58

3.1 Intervenções Humanitárias 58

3.2 As Nações Unidas e intervenções 66

3.2.1 Operações de Paz $\quad 74$

3.3 O conceito de Responsabilidade de Proteger 81

3.3.1 Introduzindo a soberania como responsabilidade $\quad 81$

3.3.2 O relatório da ICISS e as origens da Responsabilidade $\quad 84$

de Proteger

3.3.3 A relação entre Responsabilidade de Proteger, direito de 97

ingerência, intervenção humanitária e segurança humana

3.3.4 As Nações Unidas e a Responsabilidade de Proteger 100

3.3.4.1 A implementação da Responsabilidade de Proteger $\quad 114$

nas operações de paz: a Responsabilidade de Proteger e a

proteção de civis

3.3.5 Os desafios à Responsabilidade de Proteger

117

3.4 Intervenções e o contexto regional da América Latina 122

3.4.1 O contexto normativo regional 122

3.4.1.1 As normas regionais e a Organização dos Estados 136

Americanos

3.4.1.2 A não intervenção e os direitos humanos na América $\quad 141$ Latina

3.4.1.3 O posicionamento latino-americano perante a Responsabilidade de Proteger 
4. A política externa brasileira 155

4.1 A política externa brasileira e as normas: uma discussão 155 conceitual

4.2 A política externa brasileira no período pós-Guerra Fria 160

4.3 O impacto do governo Lula na política externa 166

4.4 A importância da região na inserção internacional do Brasil 170

4.5 A dimensão de segurança da política externa brasileira $\quad 177$

4.6 Brasil e intervenções $\quad 180$

4.6.1 A posição do Brasil perante a não intervenção, $\quad 180$ soberania e uso da força

4.6.1.1 A posição brasileira no período pós-Guerra Fria 184

4.6.1.2 A posição do Brasil perante as operações de paz e $\quad 198$ intervenções da ONU

4.6.1.2.1 Discursos dos representantes brasileiros na ONU 218 sobre temas relacionados a operações de paz e intervenção

4.6.2 A posição do Brasil perante a Responsabilidade de 225

Proteger

5. O Brasil e o Haiti: da década de 1990 até a MINUSTAH 229

5.1 A crise haitiana da década de 1990

5.2 A posição brasileira perante a crise haitiana nos anos 232 1990

5.3 O Brasil, a MINUSTAH e o Haiti 241

5.3.1 A MINUSTAH 241

5.3.2 O Brasil e o envolvimento no Haiti 251

5.3.2.1 A dimensão regional do envolvimento brasileiro 267

5.3.2.2 A dimensão ético solidária do envolvimento brasileiro 276 no Haiti

5.3.2.2.1 Introduzindo o debate teórico sobre política externa 276 ética

5.3.2.2.2 A boa cidadania internacional aplicada ao Brasil 282

6. Conclusão 293

7. Referências Bibliográficas 303

$\begin{array}{ll}\text { Anexos } & 337\end{array}$

1. Discursos brasileiros perante a Assembleia Geral da ONU 337 (1988-2009)

2. Lista de participantes e Sumário da mesa-redonda sobre $\quad 350$ Responsabilidade de Proteger na América Latina, em Santiago do Chile

3. Tabelas referentes à distribuição regional da contribuição para a MINUSTAH 


\section{Lista de siglas}

ACISO - Ação Cívico-Social

ALCA - Área de Livre-Comércio das Américas

ALCOPAZ - Associação Latino-Americana de Centros de Treinamento para Operações de Paz

BINUB - United Nations Integrated Office in Burundi (Bureau intégré des Nations Unies au Burundi)

CAECOPAZ - Centro Argentino de Entrenamiento Conjunto para Operaciones de Paz

CARICOM - Comunidade do Caribe (Caribbean Community)

CECOPAC - Centro Conjunto para Operaciones de Paz de Chile

CDS - Conselho de Defesa Sul-Americano

Cl Op Paz - Centro de Instrução de Operações de Paz (Brasil)

COTER - Comando de Operações Terrestres (Brasil)

CS - Conselho de Segurança (ONU)

DPKO - Department of Peacekeeping Operations - UN (Departamento de Operações de Manutenção da Paz - ONU)

ECEME - Escola de Comando e Estado Maior do Exército (Brasil)

EGN - Escola de Guerra Naval (Brasil)

ESG - Escola Superior de Guerra (Brasil)

EUA - Estados Unidos da América

FAIBRÁS - Força Armada Interamericana do Brasil

FHC - Fernando Henrique Cardoso

FIP - Força Interamericana de Paz

G-77 - Grupo dos 77

IAPTC - International Association of Peacekeeping Training Centres

IBAS - Fórum de Diálogo Índia-Brasil-África do Sul 
ICISS - Comissão Internacional sobre Intervenção e Soberania Estatal (International Commision on Intervention and State-Sovereignty)

ICRtoP - International Coalition for the Responsibility to Protect

INTERFET - International Force for East Timor (Força Internacional para o Timor Leste)

Mercosul - Mercado Comum do Sul

MICIVIH - International Civilian Mission in Haiti (OEA/ONU) (Missão Civil Internacional no Haiti)

MD - Ministério da Defesa (Brasil)

MICAH - United Nations International Civilian Support Mission in Haiti (Missão internacional de Apoio Civil ao Haiti - ONU)

MIF - Multinational Interim Force - Haiti

MINUSTAH - Mission des Nations Unies pour la Stabilisation en Haïti (Missão de Estabilização das Nações Unidas no Haiti )

MIPONUH - United Nations Civilian Police Mission in Haiti (Missão de Polícia Civil das Nações Unidas no Haiti)

MRE - Ministério das Relações Exteriores (Brasil)

NAM - Movimentos dos Não Alinhados (Non Aligned Movement)

ONU - Organização das Nações Unidas

OEA - Organização dos Estados Americanos

ONUMOZ - Operação das Nações Unidas em Moçambique (United Nations Operation in Mozambique)

OTAN - Organização do Tratado do Atlântico Norte

SRSG - Special Representative of the Secretary-General (Representante Especial do Secretário-Geral)

SUDENE - Superintendência de Desenvolvimento do Nordeste (Brasil)

UNASUL - União Sul-Americana de Nações

UNAMA - United Nations Assistance Mission in Afghanistan (Missão de Assistência das Nações Unidas no Afeganistão)

UNAVEM - III Missão de Verificação das Nações Unidas em Angola III (United Nations Verification Mission III) 
UNITAR - United Nations Institute for Training and Research

UNMIH United Nations Mission in Haiti (Missão das Nações Unidas no Haiti)

UNMIK - United Nations Mission in Kosovo (Missão das Nações Unidas em Kosovo)

UNMISET- United Nations Mission of Support in East Timor (Missão de Apoio das Nações Unidas no Timor Leste)

UNSMIH - United Nations Support Mission in Haiti (Missão de Apoio das Nações Unidas no Haiti)

UNTMIH - United Nations Transition Mission in Haiti (Missão de Transição das Nações Unidas no Haiti) 


\section{Lista de tabelas}

Tabela 1 - Contribuição latino-americana para missões de paz da ONU (p. 131)

Tabela 2 - Participação latino-americana no Conselho de Segurança da ONU (p.135)

Tabela 3 - Países contribuintes com tropas para a MINUSTAH (p. 242)

Tabela 4 - Distribuição por país de oficiais servindo no Estado-Maior da MINUSTAH (p. 244)

Tabela 5 - Discursos na Assembleia Geral (p. 337)

Tabela 6 - Contruições para a MINUSTAH por região (p. 356)

Tabela 7 - Contribuição de tropas e policiais para a MINUSTAH separados por região da América Latina e Caribe (p. 356)

Tabela 8 - Percentual de contribuição para a MINUSTAH em relação ao envolvimento total em missões de paz da ONU (p. 357) 
Quando você for convidado pra subir no adro

Da fundação casa de Jorge Amado

Pra ver do alto a fila de soldados, quase todos pretos

Dando porrada na nuca de malandros pretos

De ladrões mulatos e outros quase brancos

Tratados como pretos

Só pra mostrar aos outros quase pretos

(E são quase todos pretos)

$\mathrm{E}$ aos quase brancos pobres como pretos

Como é que pretos, pobres e mulatos

E quase brancos quase pretos de tão pobres são tratados

E não importa se os olhos do mundo inteiro

Possam estar por um momento voltados para o largo

Onde os escravos eram castigados

E hoje um batuque um batuque

Com a pureza de meninos uniformizados de escola secundária

Em dia de parada

E a grandeza épica de um povo em formação

Nos atrai, nos deslumbra e estimula

Não importa nada:

Nem o traço do sobrado

Nem a lente do fantástico,

Nem o disco de Paul Simon

Ninguém, ninguém é cidadão

Se você for a festa do pelô, e se você não for

Pense no Haiti, reze pelo Haiti

O Haiti é aqui

O Haiti não é aqui

E na TV se você vir um deputado em pânico mal dissimulado

Diante de qualquer, mas qualquer mesmo, qualquer, qualquer

Plano de educação que pareça fácil

Que pareça fácil e rápido

E vá representar uma ameaça de democratização

Do ensino do primeiro grau

E se esse mesmo deputado defender a adoção da pena capital

E o venerável cardeal disser que vê tanto espírito no feto

E nenhum no marginal

E se, ao furar o sinal, o velho sinal vermelho habitual

Notar um homem mijando na esquina da rua sobre um saco

Brilhante de lixo do Leblon

E quando ouvir o silêncio sorridente de São Paulo

Diante da chacina

111 presos indefesos, mas presos são quase todos pretos

Ou quase pretos, ou quase brancos quase pretos de tão pobres

E pobres são como podres e todos sabem como se tratam os pretos

E quando você for dar uma volta no Caribe

E quando for trepar sem camisinha

E apresentar sua participação inteligente no bloqueio a Cuba

Pense no Haiti, reze pelo Haiti

O Haiti é aqui

O Haiti não é aqui

Haiti

Caetano Veloso e Gilberto Gil 